\title{
Re-activation of the p53 pathway inhibits in vivo and in vitro growth of hormone-dependent human breast cancer cells
}

\author{
YAYUN LIANG ${ }^{1}$, CYNTHIA BESCH-WILLIFORD ${ }^{2}$, INDIRA BENAKANAKERE ${ }^{1}$ and SALMAN M. HYDER ${ }^{1,3}$ \\ ${ }^{1}$ Dalton Cardiovascular Research Center, ${ }^{2}$ Department of Veterinary Pathology and \\ ${ }^{3}$ Department of Biomedical Sciences, University of Missouri, Columbia, MO 65211, USA
}

Received April 4, 2007; Accepted May 9, 2007

\begin{abstract}
Mutations in wild-type p53 (wtp53) protein lead to loss of its tumor suppressor function in breast cancer cells, facilitating uncontrolled tumor growth. Consequently, procedures to repair defective p53 functions in tumor cells are being actively pursued. We sought to determine whether expression of wtp53 protein, or conversion of endogenous mutant p53 (mtp53) into a functional p53 protein with small molecule PRIMA-1, can override the tumor-promoting effects of naturally occurring mtp53 protein in hormone-responsive T47-D human breast cancer cells. We show that transfection of wtp53 gene into T47-D cells suppresses their proliferation in regular media, and inhibits estrogen-dependent cell proliferation in media containing dextran-coated charcoal treated serum. Growth inhibition was not due to the absence of estrogen receptor- $\alpha$ or estrogen receptor- $\beta$ though receptor levels for estrogen receptor- $\alpha$ were drastically reduced in wtp53 expressing cells. Focused microarray analysis of wtp53 expressing cells revealed suppression of PCNA cell-cycle regulatory mRNA and protein. Wild-type p53 transfected T47-D cells also failed to grow in vivo in estrogen supplemented nude mice. Furthermore, xenografts obtained with parental T47-D cells expressing mtp53 grew poorly in nude mice treated with PRIMA-1. PRIMA-1 treated tumors exhibited
\end{abstract}

Correspondence to: Dr Salman M. Hyder, Dalton Cardiovascular Research Center, University of Missouri-Columbia, 134 Research Park Drive, Columbia, MO 65211, USA

E-mail: hyders@missouri.edu

Abbreviations: DCC, dextran-coated charcoal; ELISA, enzymelinked immunosorbent assay; ER, estrogen receptor; FBS, fetal bovine serum; Ki67, proliferation marker; MPA, medroxyprogesterone acetate; mt, mutant; PCDNA, parental T47-D cells transfected with backbone vector; PCNA, proliferating cell nuclear antigen; p53, 53-kDa tumor suppressor protein; p53-L, T47-D cells transfected with wild-type p53 expression plasmid; PR, progesterone receptor; SRB, sulforhodamine B; VEGF, vascular endothelial growth factor; wt, wild-type

Key words: p53, estrogen, breast cancer, nude mice, PRIMA-1 a low proliferation index, even though mice were estrogensupplemented. PRIMA-1 treatment of tumor cells suppressed VEGF and induced expression of estrogen receptor- $\beta$ though expression of estrogen receptor- $\alpha$ and progesterone receptors was unaffected. These data indicate that alteration of the p53 signal transduction pathway by re-expression of wtp53 protein in T47-D cells, or treatment of parental cells with PRIMA-1, can prevent in vivo and in vitro proliferation of T47-D breast cancer cells.

\section{Introduction}

p53 is a multifunction tumor suppressor protein that is a key player in the cellular response to DNA damage and other types of cellular stress $(1,2)$. Activated wild-type p53 (wtp53) plays a key role in signaling pathways that lead to cellular stress-induced cell cycle arrest and/or apoptosis. Mutations in wtp53 are frequent in a wide range of cancers, including breast cancer, and this likely contributes to the uncontrolled growth phenotype associated with tumor cells $(1,2)$. Many mutant p53 alleles in cancer cells express dysfunctional mutant p53 protein $(\mathrm{mtp} 53)$ at a high level $(3,4)$. Approximately $50 \%$ of all breast cancers carry point mutations in the p53 gene, and the majority of mtp53 alleles in breast cancer cells are defective in DNA binding as well as in cell cycle checkpoints and DNA damage-induced induction of apoptosis $(5,6)$. Mutations in p53 or the p53 pathway are thought to play a key role in promoting tumor cell survival and tumor cell resistance to chemotherapeutic drugs $(7,8)$.

PRIMA-1 is a small molecule that activates the mtp53 protein and restores wild-type functions of this protein $(9,10)$. For example, PRIMA-1 stimulates expression of p21 and other p53-dependent promoters in several breast cancer cells that express mtp53 (10), and suppresses hormone-induced vascular endothelial growth factor (VEGF) expression (10). VEGF is associated with angiogenesis in tumor tissue $(11,12)$. Because PRIMA-1 at least partially restores wtp53 function in several breast cancer cells, it seemed possible that coexpression of wtp53 and/or treatment with PRIMA-1 might have therapeutic potential as an anti-cancer/anti-angiogenic treatment for hormone responsive breast cancer cells that express mtp53 protein by suppressing the production of VEGF. The premise of this strategy is that wtp53 protein is essentially dominant over endogenous mtp53 in cancer cells. This study tests the hypothesis that growth of hormone- 
responsive T47-D human breast cancer cell line can be suppressed by co-expression of wtp53 or by treatment with PRIMA-1. The results suggest that reactivation of the p53 mediated pathways may have potential as an anti-cancer strategy for human breast cancer and possibly other cancers.

\section{Materials and methods}

Materials. T47-D cells expressing mtp53 were from ATCC (Manassas, VA). Phenol red-free DMEM/F12 medium, phosphate-buffered saline, and $0.05 \%$ trypsin-EDTA were from Invitrogen Corporation \& Life Technologies (Grand Island, $\mathrm{NY}$ ) and fetal bovine serum (FBS) was from JRH Biosciences (Lenexa, KS). PRIMA-1 (p53-reactivation and induction of massive apoptosis) was from Tocris (Ellisville, MO). 17-ßestradiol (1.7 mg/pellet, 60-day release) and placebo pellets were from Innovative Research of America (Sarasota, FL). sulforhodamine B (SRB) was from Sigma (St. Louis, MO), BD Matrigel Matrix was from BD Biosciences (Bedford, MA), anti-PCNA antibody was from Dako (Carpinteria, CA) and anti-ER- $\alpha$ and anti-ER- $\beta$ antibodies for Western blots were from Santa Cruz Biotechnology, Inc. (Santa Cruz, CA). The plasmid with the p53 gene cloned into pcDNA vector was a kind gift from Dr Xinbin Chen, University of Alabama.

Cell lines and cell culture. T47-D cells were stably transfected with a wtp53 expression plasmid or vector (PCDNA), selected for neomycin resistance and tested for expression of functional p53 as described previously (10). Stably transfected T47-D cells were selected for loss of progestin-dependent induction of VEGF induction (10). One such cell line, T47-D-p53-L (referred to as p53-L cells), and a control cell line, T47-DPCDNA-A (referred to as PCDNA-A), carrying vector DNA, were selected for further study. Cells were maintained in phenol red-free DME/F12 medium supplemented with $10 \%$ FBS (T47-D) or the same medium supplemented with $200 \mu \mathrm{g} / \mathrm{ml} \mathrm{G} 418$ for transfected clones. All cells were grown in $100 \times 20 \mathrm{~mm}$ tissue culture dishes and harvested with $0.05 \%$ trypsin-EDTA.

Cell proliferation assay by SRB. A sulforhodamine B (SRB) assay was used to measure cell viability, as previously described $(13,14)$. Briefly, T47-D, PCDNA-A, and p53-L cells were grown to $70 \%$ confluence, after which media were replaced with 5\% FBS-DCC DMEM/F12 and incubated for $24 \mathrm{~h}$ to deplete any endogenous steroids. Cells were harvested by trypsin-EDTA treatment and $4 \times 10^{3}$ cells/well were seeded in 96-well plates in $100 \mu \mathrm{l} 5 \%$ FBS-DCC DMEM/F12 and incubated overnight at $37^{\circ} \mathrm{C}$ with $5 \% \mathrm{CO}_{2}$. Cells were washed once with DMEM/F12 and incubated for $48 \mathrm{~h}$ in $100 \mu 15 \%$ FBS-DCC DMEM/F12 in the presence of a variable concentration of estradiol-17ß. Surviving or adherent cells were fixed in situ by adding $100 \mu 150 \%$ cold trichloroacetic acid for $1 \mathrm{~h}$ at $4^{\circ} \mathrm{C}$. Cells were washed with ice-water, dried and stained with $50 \mu 14 \% \mathrm{SRB}$ for $8 \mathrm{~min}$ at room temperature. Unbound stain was removed by washing cells $5 \mathrm{X}$ with cold $1 \%$ acetic acid and dried at room temperature. Cell bound stain was solubilized in $150 \mu 110 \mathrm{mM}$ Tris-buffer and quantified at $520 \mathrm{~nm}$ using a SpecTRA MAX 190 microplate reader (Sunnyvale, CA). Six-wells were used for each dose/experi- mental condition and each experiment was performed at least twice.

Preparation of nuclear extracts. A nuclear extract kit was used according to the manufacturer's instructions (Active Motif, Carlsbad, CA). Briefly, cells were grown in 100-mm dishes, washed with $3 \mathrm{ml}$ ice-cold PBS containing phosphatase inhibitors and harvested by gentle scraping with a cell lifter. Cells were centrifuged for $5 \mathrm{~min}$ at $200 \mathrm{x} \mathrm{g}$ at $4^{\circ} \mathrm{C}$, re-suspended in $500 \mu 11 \mathrm{X}$ hypotonic buffer (Active Motif kit) and incubated for $15 \mathrm{~min}$ on ice. Detergent $(25 \mu \mathrm{l})$ was added and the extract was centrifuged for $30 \mathrm{sec}$ at $14,000 \mathrm{x} \mathrm{g}$ at $4^{\circ} \mathrm{C}$. The pellet was re-suspended in $50 \mu \mathrm{l}$ complete lysis buffer containing $1 \mathrm{mM}$ DTT and 1\% protease inhibitor cocktail and incubated on ice for $30 \mathrm{~min}$ with shaking. Samples were centrifuged at $14,000 \mathrm{x} \mathrm{g}$ for $15 \mathrm{~min}$, and the supernatant was transferred to a microcentrifuge tube, aliquoted, and stored at $-80^{\circ} \mathrm{C}$. Supernatant was adjusted to the desired protein concentration prior to use.

Immunoblotting. Nuclear extracts (35 $\mu \mathrm{g}$ per lane) were separated on a NuPAGE $10 \%$ Bis-Tris Gel (Invitrogen, Carlsbad, CA). Electrophoresis was performed at $100 \mathrm{~V}$ for 2 h using NuPAGE MES-SDS Running Buffer. Separated proteins were electro-transferred to a polyvinylidene difluoride membrane (Bio-Rad Laboratories, Hercules, CA) at $35 \mathrm{~V}$ for $1.5 \mathrm{~h}$. The blots were blocked for $1 \mathrm{~h}$ at room temperature (RT) in 5\% non-fat dry milk in TBS containing 0.1\% Tween-20 (TBS-T), and incubated with primary antibody for $2 \mathrm{~h}$ at RT at the indicated dilution: PCNA (clone PC10; 1:800 dilution), anti-PR (1:300 dilution of AB-52), anti-ER $\alpha$ (D-12; 1:200 dilution), ant-ER- $\beta$ (H-150; 1:200 dilution). The blots were washed 3 times with TBS-T, incubated with secondary antibody for $1 \mathrm{~h}$ at RT, and washed 6 times ( 8 min each) with TBS-T. Immuno-reactive bands were visualized using the ECL plus detection kit (Amersham, Pharmacia Biotech, Arlington Heights, IL). Membranes were stripped and reblotted for $\beta$-actin (Sigma). Immuno-reactive protein signal was normalized to the actin signal in the same sample.

Animal xenograft tumor studies in nude mice with wtp53 transfected cells. Female athymic nu/nu nude mice, 5-6-weeks old (18-22 g) were purchased from Harlan Sprague Dawley, Inc. (Indianapolis, IN). The mice were housed in a laminar air-flow cabinet under specific pathogen-free conditions. All facilities were approved by the American Association for Accreditation of Laboratory Animal Care in accordance with the current regulations and standards of the United States Department of Agriculture, the Department of Health and Human Services, and the NIH. All procedures were approved by IACUC.

Nude mice were inoculated in the dorsal flank with a 17- 3 estradiol pellet $(1.7 \mathrm{mg} /$ pellet; 60 -day release) $48 \mathrm{~h}$ prior to injection with tumor cells. PCDNA-A and p53-L cells were harvested by trypsinization, washed twice with DMEM/F12, re-suspended in $100 \mu 1 \mathrm{DMEM} / \mathrm{F} 12$ and injected $\left(1 \times 10^{7}\right.$ cells per mouse) subcutaneously in the dorsal flank. Tumors were measured every 3 days with a digital caliper and tumor volumes were calculated using the following formula: ( $\mathrm{L} \mathrm{x} \mathrm{W} \mathrm{x} \mathrm{H)} \mathrm{x}$ $\pi / 6$ (15). 
PRIMA-1 treatment of xenografts obtained from parental T47-D cells expressing mtp53 protein. Mice were inoculated with estradiol pellets as described above. After $48 \mathrm{~h}, 5 \times 10^{6}$ T47-D cells were re-suspended in $100 \mu 1$ DMEM/F12 medium mixed with Matrigel $(50 \% \mathrm{v} / \mathrm{v})$, and inoculated into both flanks subcutaneously. Animals were randomly assigned to two groups of 5 mice each. One group of mice received $25 \mathrm{mg} /$ $\mathrm{kg}$ /day PRIMA-1 by i.p injection $2 \mathrm{~h}$ prior to injection of tumor cells (details in Fig. 5A). This treatment was continued for two additional days and then PRIMA-1 treatment was continued at $50 \mathrm{mg} / \mathrm{kg} /$ day by tail vein injection for 10 days. Experiments were terminated by sacrificing animals at $6 \mathrm{~h}$ after the final PRIMA-1 injection. Tumors were measured every 2 days with digital calipers and harvested into $4 \%$ paraformaldehyde for future studies. Tumor volume was calculated using the formula ( $\mathrm{L} \times \mathrm{W} \times \mathrm{H}) \times \pi / 6(15)$.

Histology and immunohistochemical analysis. Immunohistochemical analysis was carried out for Ki67, VEGF, Factor VIII, PR, ER- $\alpha$ and ER- $\beta$. Tumor tissues was fixed overnight in $4 \%$ paraformaldehyde for immunohistochemistry. Tissues were processed for paraffin infiltration and embedding. Five- $\mu \mathrm{m}$ sections were mounted onto ProbeOn Plus microscope slides (Fisher Scientific Inc., Pittsburgh, PA), stained with hemotoxylin-eosin and examined for cellularity by light microscopic analysis. For immunohistochemical analysis, sections were de-waxed in xylene, rehydrated through graded concentrations of ethanol, then rinsed in distilled water, and, if necessary, stored in phosphate-buffered saline (PBS) at $4^{\circ} \mathrm{C}$ until use. Sections were subjected to heat-induced epitope retrieval in $10 \mathrm{mM}$ citrate buffer (pH 6.0) (VEGF, $\mathrm{Ki67}, \mathrm{ER}, \mathrm{PR})$ or proteinase $\mathrm{K}(20 \mu \mathrm{g} / \mathrm{ml}$ in TE buffer, $\mathrm{pH} 8.0)$ (Factor VIII antigen). Slides were treated with $3 \%$ hydrogen peroxide in absolute methanol (to inactivate endogenous peroxidase activity), washed in $3 \mathrm{X}$ PBS, incubated in blocking buffer with $5 \%$ bovine serum albumin for $20 \mathrm{~min}$ and treated with polyclonal antibody at room temperature for $60 \mathrm{~min}$ at the following dilution: anti-VEGF antibody [1:200 dilution of a rabbit anti-VEGF polyclonal antibody (sc-152); Santa Cruz Biotechnology, Inc.]; anti-Ki67 [1:200 dilution of rabbit anti-Ki67 polyclonal antibody (RB 1510-P); Lab Vision, Fremont, CA]; anti-factor VIII antibody [1:400 dilution of a rabbit anti-factor VIII polyclonal antibody (A0082); Dako], anti-PR [1:50 dilution of a rabbit anti-PR polyclonal antibody, (A0098); Dako], anti-ER $\alpha$ [1:300 dilution of a rabbit anti-ER $\alpha$ polyclonal antibody (sc-542); Santa Cruz Biotechnology, Inc.], and anti-ER- $\beta$ [1:50 dilution of a mouse anti-ER- $\beta$ monoclonal antibody (MCA1974s); Serotec, Inc., Raleigh, NC]. Sections were then washed and sequentially incubated with a secondary antibody (biotinylated swine anti-mouse $\mathrm{IgG}$ and biotinylated swine anti-rabbit IgG; Dako), and a streptavidin-linked horseradish peroxidase product (Dako) for $30 \mathrm{~min}$, also at room temperature. Alternatively, some sections were incubated with EnVision ${ }^{+}$, a horseradish peroxidase labeled polymer conjugated with anti-rabbit antibodies (Dako). Bound antibodies were visualized following incubation with 3, 3'-diaminobenzidine tetrahydrochloride ( $0.05 \%$ with $0.015 \% \mathrm{H}_{2} \mathrm{O}_{2}$ in PBS) solution (Dako) for 3-5 min. Sections were counterstained with Mayer's hematoxylin, dehydrated, cleared and coverslipped for microscopic examination.
Proliferation index. The number of Ki67 positively staining nuclei was counted per $\sim 1000$ tumor nuclei in the most intensely stained areas of tumor sections. We measured areas that were uniform $\left(2049 \mu^{2}\right)$ among samples. The proliferation index was then defined as Ki67 positive nuclei/Ki67 positive $+\mathrm{Ki} 67$ negative nuclei $\mathrm{x} 100$.

RNA isolation and synthesis of cDNA. Total RNA was isolated using Ultra spec RNA reagent (Biotecx Products, Oxon, UK). Total RNA was used as a template for biotinylated probe synthesis using TrueLabeling-AMP Kit (SuperArray, Inc., Bethseda, MD) according to the manufacturer's instructions.

Hybridization and chemiluminescent detection. Microarray membranes were hybridized using the Oligo GEArray Kit (SuperArray, Inc.) according to the manufacturer's instructions. The chemiluminescent detection kit (SuperArray, Inc.) was used to conform proper probe hybridization. Membranes were washed, rinsed, incubated with ECL chemiluminescent substrate (Amersham, Arlington Heights, IL), after which the membranes were exposed to radiographic film. All images were stored electronically and uploaded into an integrated (web-based) GEArray Expression Analysis System at the SuperArray web site. Data analysis and normalization was based on quartile analysis as described by SuperArray Inc.

Statistical analysis. Statistical significance was measured using Student's t-test or One-way analysis of variance (ANOVA) with repeated measure over time. When necessary, it was assumed that ANOVA was non-parametric. Values are reported as mean \pm SE. For samples with significant F-ratio $(p<0.05)$, the Student-Newman-Keuls multirange test was employed (SigmaStat).

\section{Results}

Stable T47-D transfectants that co-express wtp53 and mtp53 were selected by screening for neomycin resistance, the presence of wtp53 protein and for inducibility of p21 as previously described (10). Stable transfectants were also screened for progesterone-stimulated induction of VEGF and expression of p53 was found to suppress hormoneinduced VEGF in several clones (10). A cell line that lacks progesterone-inducible VEGF, p53-L, and a control cell line carrying empty vector, PCDNA-A, were selected for further study. Cell proliferation studies were carried out on p53-L cells in the presence of $10 \%$ FBS using a sulforhodamine B (SRB) dye-binding assay $(13,14)$. Control cells included PCDNA-A cells and parental T47-D cells. The results showed that p53-L cells grew 1.5- to 2-fold less efficiently than T47-D or PCDNA-A cells in the presence of $10 \%$ FBS. These data suggest that stable co-expression of wtp53 protein suppresses in vitro proliferation of T47-D cells in the presence of serum.

The expression of human cell-cycle related genes were compared in p53-L and PCDNA-A cells using a human gene array profiling system (SuperArray). The results showed that PCNA mRNA was the most extensively down-regulated (2.3-fold) in p53-L compared with expression in PCDNA-A cells, and correspondingly PCNA protein was 2-fold lower in 


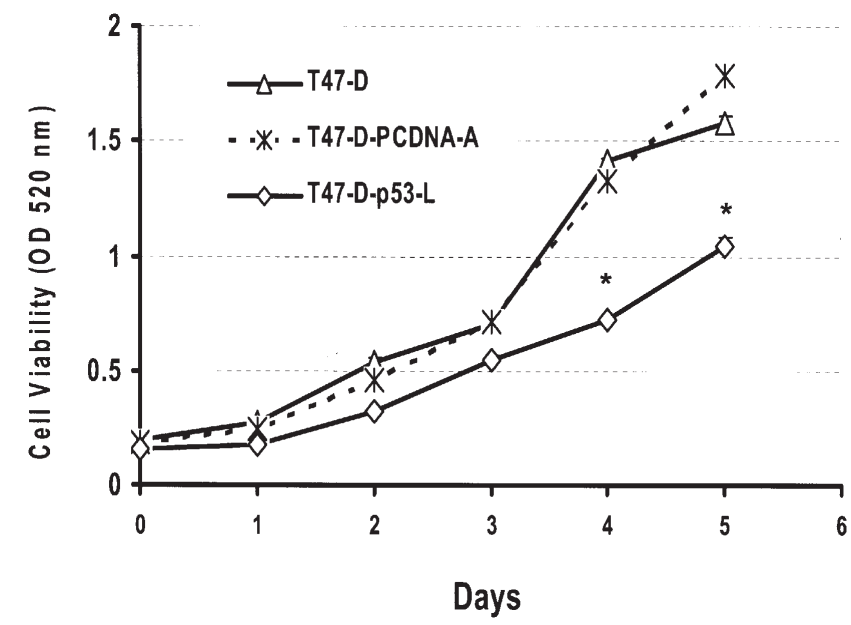

Figure 1. Growth of p53-L, PCDNA-A and T47-D cells. p53-L, PCDNA-A and T47-D cells $\left(4 \times 10^{3}\right.$ cells/well) were seeded into a 96-well plate and incubated overnight in $100 \mu 1 \mathrm{DMEM} / \mathrm{F} 12+10 \%$ FBS. Media was replaced and cells were incubated for the indicated period of time with an additional media change on day 3 . Cells were fixed in situ and cell viability was determined from SRB absorbance (see Materials and methods). Asterisks indicate values that are significantly different than control ( $\mathrm{p}<0.05$, ANOVA).

p53-L cells than in PCDNA-A and T47-D cells (Fig. 2A and $\mathrm{B}$ ).

Estrogen stimulates proliferation of many breast cancer cell lines including T47-D cells (16) thus, the effect of exogenous estrogen on growth of p53-L, PCDNA-A and T47-D cells was examined by measuring cell proliferation as described above. The results showed that exogenous estradiol-17- $B$ $(0.1 \mathrm{nM}$ to $1 \mu \mathrm{M})$ strongly stimulated growth of PCDNA-A and T47-D cells in a dose-dependent manner, but did not stimulate the growth of p53-L cells (Fig. 3A), despite continued expression of estrogen receptor- $\alpha$ ER- $\alpha$ ) in these cells albeit at 3-4-fold lower level in p53-L cells than in PCDNA-A and T47-D cells. The level of ER- $B$ was similar in p53-L, PCDNA-A and T47-D cells (Fig. 3B).

The proliferative capacity of p53-L cells was also examined in nude mice treated with estradiol pellets (note that T47-D cells do not grow as xenografts in nude mice in the absence of exogenous estrogen). p53-L and PCDNA-A cells were injected subcutaneously in the left and right dorsal flanks of nude mice, and tumor volume was measured by caliper every three days for 30 days. The results showed that mice injected with PCDNA-A cells formed large tumors, which grew for $\sim 10$ days before beginning to regress (Fig. 4). Tumors of similar size formed with similar efficiency in mice injected with T47-D parental cells (not shown). In contrast, mice injected with p53-L cells formed very small tumors by day 10 , which regressed slowly from 10 to 30 days post-injection (Fig. 4). These data demonstrate that co-expression of wtp53 suppresses growth of T47-D cells in vivo in nude mice supplemented with exogenous estrogen.

The studies described above demonstrate that co-expression of wtp53 and mtp53 in T47-D cells suppresses cell growth in vivo and in vitro. This result suggests that cancer cell growth might also be suppressed by restoring wild-type function to mtp53. This approach was tested here using PRIMA-1, a previously characterized small molecule activator of mtp53 (9). For this experiment, estrogen pellets were implanted
$\underline{A}$

\section{Human cell cycle array}
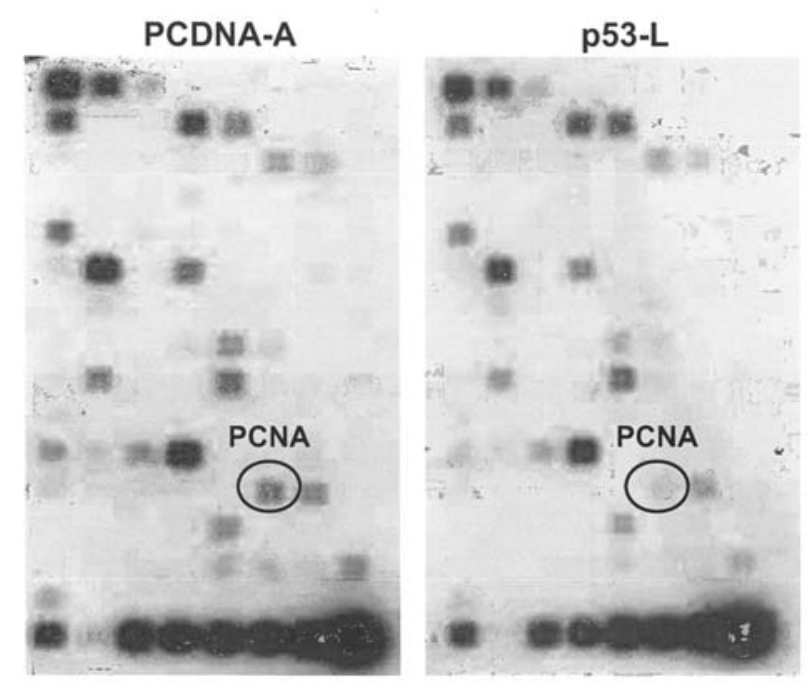

$\underline{B}$

T47-D PCDNA-A p53-L

PCNA

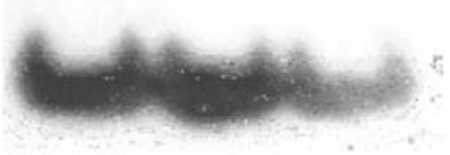

B-Actin
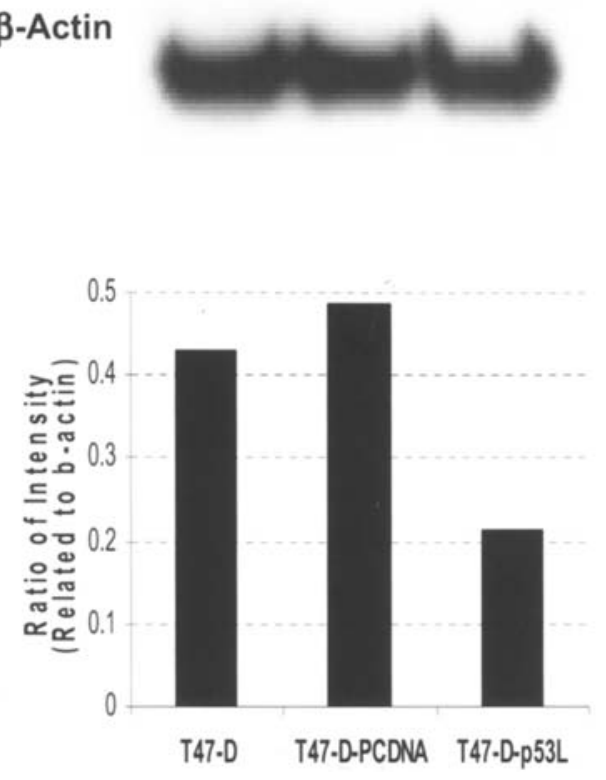

Figure 2. (A) Gene expression analysis in PCDNA-A and p53-L cells. Gene expression was analyzed using a human SuperArray microchip for cell cycle genes as described in Materials and methods. Signal intensity was normalized using an interquartile approach and the GEArray expression analysis suite from SuperArray. Changes in expression $\geq 2$. 0 -fold were assessed. Only PCNA was found to reduce by $>2$-fold in the p53L cells (circles). (B) Western blotting of PCNA in T47-D, PCDNA-A and p53-L cells. Cells were grown to $80 \%$ confluence in the presence of $10 \%$ FBS, washed with cold PBS and harvested. Nuclear extract was prepared and analyzed by Western blotting using antibody to PCNA. Quantification of data is shown in bar graph to the right. Values were normalized to $B$-actin.

into nude mice, the mice were treated with PRIMA- 1 for $2 \mathrm{~h}$, and then tumor cells were mixed with Matrigel and injected subcutaneously in the left and right dorsal flanks of nude mice 


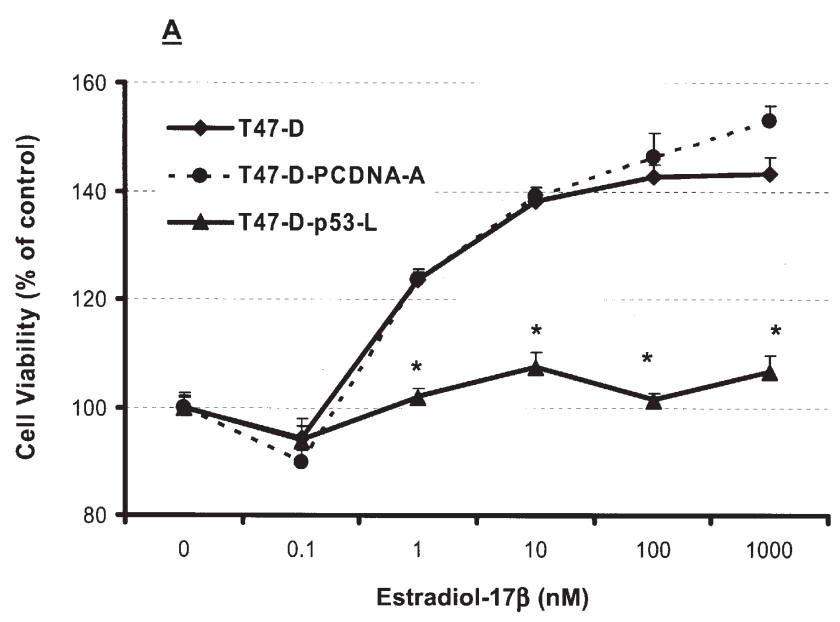

B

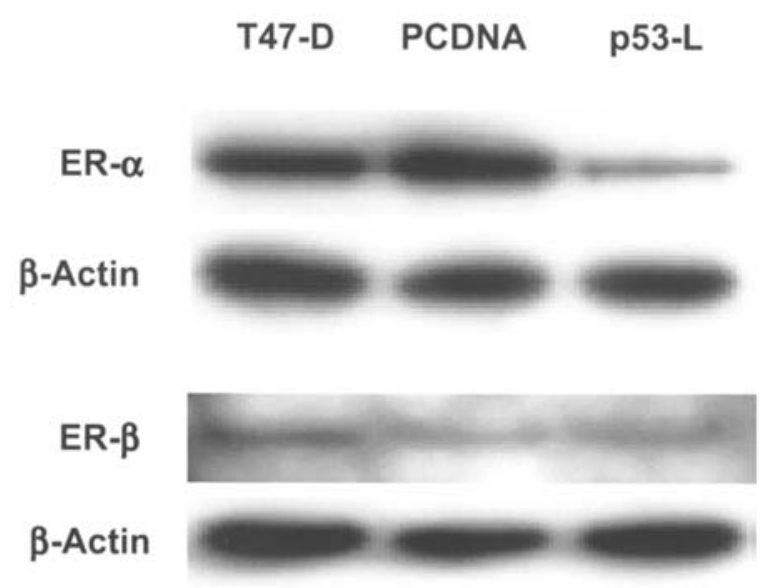

Figure 3. (A) Estrogen-dependent cell proliferation. The indicated cell lines were placed in 96-well plates in medium containing $5 \%$ charcoal-treated serum for $24 \mathrm{~h}$, washed once with DMEM/F12 medium and seeded into 96-well plates overnight with the same medium containing different concentration of 17-ß-estradiol for $48 \mathrm{~h}$. Cell viability was determined as in Fig. 1. Asterisks indicate values that are significantly different than control. (B) Western blotting of ER- $\alpha$ and ER- $\beta$ in nuclear extracts of T47-D, p53L and PCDNA-A cells.

(Fig. 5A). PRIMA-1 dosing continued for 3 days at $25 \mathrm{mg} / \mathrm{kg}$ and then for 10 days at $50 \mathrm{mg} / \mathrm{kg}$ as shown in Fig. 5A. Tumor volume was measured every other day for 14 days (Fig. 5B). The results show that PRIMA-1 strongly inhibits tumor growth in nude mice injected with T47-D cells (Fig. 5B). Treated animals showed no toxic side effects of PRIMA-1 as also reported by others (9) and maintained normal body weight over the duration of the experiment (Fig. 5C). Very small tumors were detected at the site of injection in animals treated with PRIMA-1 (Fig. 5D). These data show that PRIMA-1 strongly inhibits growth of T47-D as xenograft tumors in nude mice.

Because PRIMA-1-treated mice injected with T47-D cells developed small tumors (Fig. 5B and D), representative tumors were excised and subject to histological analysis. The results showed tumor cells in both PRIMA-1 treated mice and control mice; however, the cellularity of tumors from PRIMA-1 treated animals was sparse (Fig. 6A, left panel). There were fewer

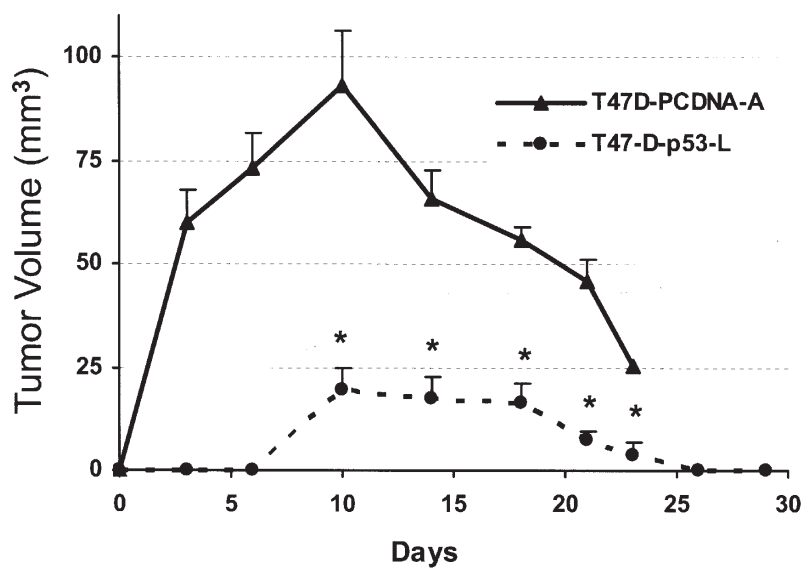

Figure 4. Xenograft tumors in nude mice derived from PCDNA-A and p53-L. Five- to 6-week old nude mice were inoculated with 17-ß-estradiol pellet (see Materials and methods). Forty-eight hours later, PCDNA-A or p53-L cells $\left(1 \times 10^{7}\right.$ cells) in $100 \mu 1 \mathrm{DMEF} / \mathrm{F} 12$ were injected subcutaneously in the left and right dorsal flanks of each mouse. Tumors were measured every 3 days with a digital caliper and tumor volume was calculated using the formula $(\mathrm{L} \times \mathrm{W} \times \mathrm{H}) \times \pi / 6$. Asterisks indicate values that are significantly different than control ( $\mathrm{p}<0.05$ using t-test).

Ki67-positive cells in tumors from PRIMA-1-treated mice than in controls (Fig. 6A, middle panel). The proliferation index was $37 \pm 5$ (mean \pm SEM) in PRIMA-1 treated samples compared with $57 \pm 4$ in the control samples; $\mathrm{p}<0.05$, Student's $\mathrm{t}$-test). The number of apoptotic cells was similar in PRIMA-1treated and control animals as determined using TUNNEL assay (not shown). These data suggest that PRIMA-1 mainly inhibited proliferation of T47-D cells in vivo under the protocol tested. Furthermore, VEGF expression was severely inhibited in tissue samples from PRIMA-1-treated animals (Fig. 6A, right panel). Analysis of Factor VIII expression and tumor vascularity was inconclusive due to insufficient tissue. PRIMA-1 did not alter the ER- $\alpha$ or progesterone receptor status of tumor tissue, but it significantly stimulated expression of ER- $\beta$ in tumor cells isolated from treated animals (Fig. 6B).

\section{Discussion}

The present study demonstrates that over-expressed wtp53 is dominant over mtp53 in T47-D cells, and that co-expression of wtp53 and mtp53 or activation of endogenous mtp53 is a viable strategy to inhibit tumor cell growth. Importantly, activation of the p53 pathway by one of these two approaches inhibits proliferation of T47-D cells in cell culture in the presence of serum-derived growth factors or exogenous estrogen, and inhibits growth of T47-D xenograft tumors in vivo in nude mice.

The results presented here show that co-expression of wtp53 protein inhibits growth of T47-D cells in culture in the presence of $10 \%$ FBS. The mechanism of this effect is not known. It is possible that wtp53 protein binds to mtp53, thereby preventing adverse biological impacts of the mutant protein. This result is consistent with recent observations from human clinical trials, showing that expression of wtp53 protein can cause regression of human breast disease many of which contain the mtp53 protein initially $(17,18)$. Alternatively, co- 
$\underline{A}$

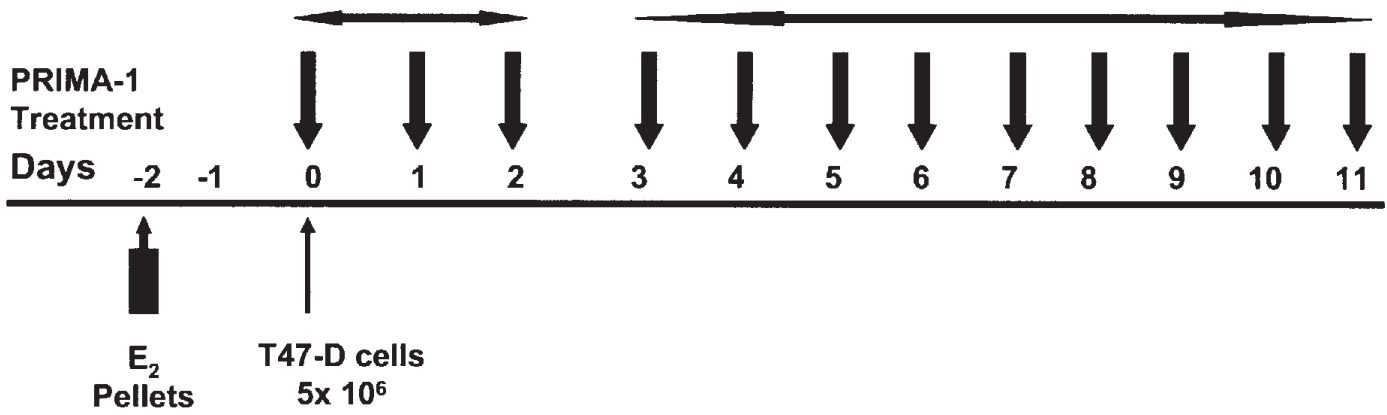



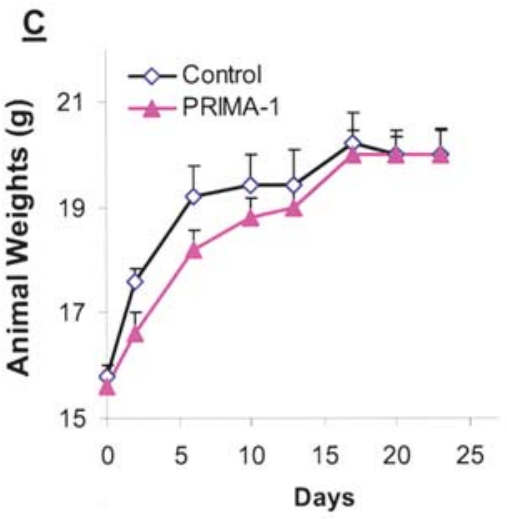

$\underline{\mathbf{D}}$
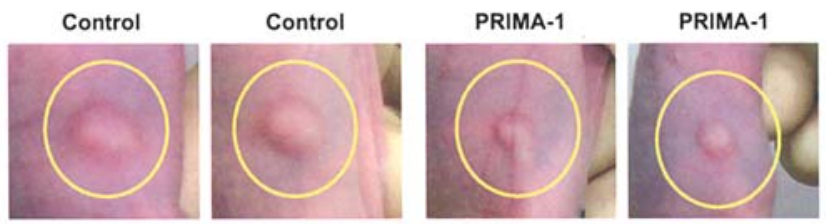

Figure 5. (A) PRIMA-1 dosing regimen is shown schematically. Dose and time of each injections or PRIMA-1 is indicated. Time of inoculation with $\mathrm{E}_{2}$ pellet and tumor cells is also indicated. (B) Xenograft tumor volume in PRIMA-1-treated and control nude mice. Five- to 6-week old nude mice were inoculated with 17-ß-estradiol pellet. Forty-eight hours later, mice were injected subcutaneously in both dorsal flanks with $5 \times 10^{6} \mathrm{~T} 47-\mathrm{D}$ cells in $100 \mu 1 \mathrm{DMEF} / \mathrm{F} 12$ containing Matrigel. PRIMA-1-treated mice were dosed as shown in (A). Tumors were measured every 2 days and tumor volume was calculated using the formula $(\mathrm{L} \times \mathrm{W} \times \mathrm{H}) \times \pi / 6$. Asterisks indicate values that are significantly different than control (p<0.05 using t-test). (C) Average body weight of PRIMA-1treated and control animals. (D) Selected images of xenograft tumors in PRIMA-1-treated and control animals at the end of the experiment as shown in (A).

expression of wtp53 in T47-D cells may directly regulate genes involved in cell cycle arrest and/or apoptosis, thus indirectly blocking the adverse growth-promoting effects of mtp53. Focused microarray studies in T47-D cells coexpressing wtp53 demonstrated decreased transcription of proliferation genes including PCNA. These data suggest that T47-D cells have an intact p53 pathway, which could potentially be exploited for therapeutic benefit. One caveat is that the present observations may not be generalized to all human breast cancer cells or to other human cancer cell types. For example, additional downstream effectors of cell cycle control may be present or absent in cell lines other than T47-D. Additional studies are needed to address this possibility. Nevertheless preliminary studies showed that co-expression of wtp53 prevents progesterone-dependent induction of VEGF not only in T47-D cells but also in two other cell lines containing mt53, namely BT-474 and HCC-1428 cells, suggesting that restoration of the $\mathrm{p} 53$ pathway is achiev- able in many different breast cancer cell types following an attempt to restore this function with wtp53 expressing plasmid or use of small molecules such as PRIMA-1 (10).

Co-expression of wtp53 in T47-D cells also blocked estrogen-dependent cell growth. Cell growth was inhibited despite normal expression of ER- $\beta$ though there was a 3 - to 4 -fold reduced expression of ER- $\alpha$. Previous studies suggest that wtp53 may also interact with and inhibit downstream signaling by ER- $\alpha$ (19-21), though the role of p53 in influencing ER- $\beta$ mediated signal transduction pathway remains unknown. Alternatively, wtp53 may suppress other signal transduction pathways (i.e., MAPK or the PI3 kinase) which in turn influence ER-dependent signaling (22).

This study also shows that co-expression of wtp53 in T47-D cells inhibits growth of xenograft tumors in nude mice. There are several possible explanations for this result. First, co-expression of wtp53 could induce inhibitors of tumor or endothelial cell growth and/or inhibitors of angiogenesis. 
$\underline{A}$



H \& E-PRIMA-1-\#25-C

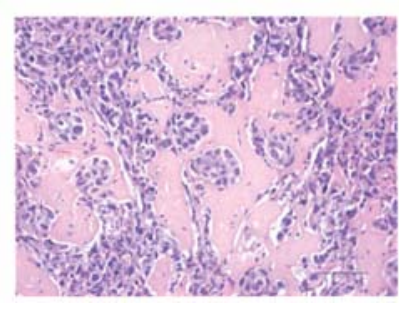

Ki67-Control-\#31

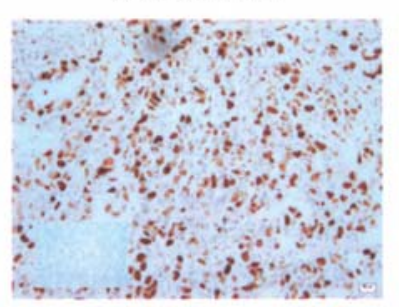

Ki67-PRIMA-1-\#25



VEGF-Control-\#31



VEGF-PRIMA-1-\#25

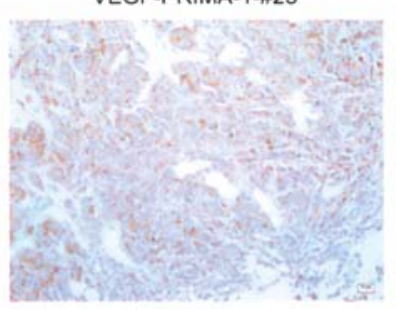

$\underline{B}$

PR

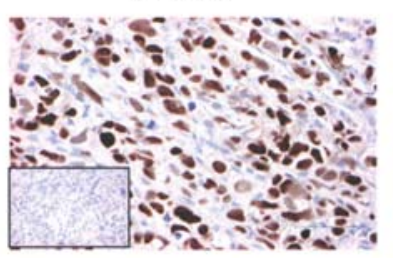

$E R \alpha$

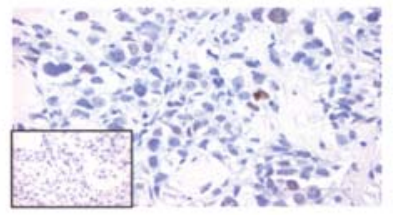

ER $\beta$

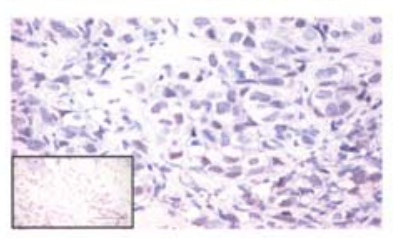

PRIMA-1
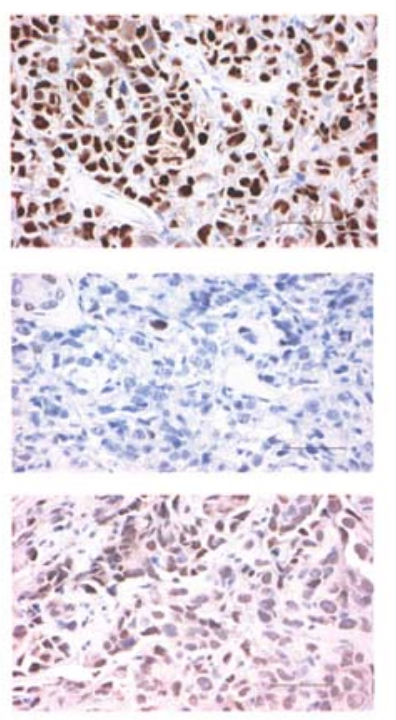

Figure 6 (A) Ki67 and VEGF expression in T47-D xenograft tumors in PRIMA-1-treated and control nude mice. Tumors were excised from PRIMA-1-treated and control nude mice and analyzed by H\&E staining or immunohistochemistry as indicated. (B) Sections from tumors shown in (A) were stained for various steroid receptors as indicated.

Second, wtp53 could promote apoptosis of T47-D cells in xenograft tumors. Third, co-expression of wtp53 inhibited proliferation and induced cell cycle arrest in T47-D cells in xenograft tumors. These possibilities are not mutually exclusive and all three mechanisms could contribute to suppression of xenograft tumors in nude mice. Additional studies will explore and possibly discriminate between one or a combination of these effects.

Activation of endogenous mtp53 in T47-D cells by PRIMA-1 also strongly inhibited, but did not completely suppress growth of T47-D xenograft tumors in nude mice. Histological analysis of residual tumor masses in PRIMA-1treated nude mice suggests that tumor development may have failed due to insufficient vascularization. This is consistent with the fact that PRIMA-1 down-regulates expression of VEGF (Fig. 6A) a potent inducer of vascularization as well as

a survival factor for breast cancer cells (23). Since PRIMA-1 activates mtp53 into its wt counterpart, it is possible that PRIMA-1 activated 553 protein blocks the production of VEGF at transcriptional level as has been shown by others $(24,25)$. As an alternative for lack of tumor growth, nascent blood vessels at the site of tumor cell injection might increase the local concentration of PRIMA-1, ultimately causing apoptosis and/or cell cycle arrest. More information on the pharmacokinetic and pharmacodynamic properties of PRIMA-1 in vivo is needed to evaluate these possibilities. Future experiments will test alternative PRIMA-1 dosing strategies and their effects on growth of T47-D xenograft tumors in nude mice. Such studies are feasible because to date, PRIMA-1 has not been associated with any adverse toxicity in nude mice (9).

Immunohistochemical analysis also showed that tumors in PRIMA-1-treated mice contain a smaller fraction of Ki67positive cells, indicating a lower rate of cell proliferation. In contrast, the rate of apoptosis was similar in tumors in PRIMA-1-treated and control mice. Since the tumors were collected at the end of the experiment it is possible that initially there was a higher apoptotic rate reducing the number of cells in tumor overall. Thus PRIMA-1 may have effects both in terms of increasing apoptosis as shown by others (9) and in reducing cellular proliferation. It is also possible that the rate of apoptosis would increase in tumors of mice exposed to a higher concentration of PRIMA-1, leading to complete suppression of xenograft tumors. Thus, future studies will include PRIMA-1 dose escalation and alternative routes of PRIMA-1 delivery.

Interestingly, expression of ER- $\beta$ increased in tumors in mice treated with PRIMA-1 (Fig. 6B), while expression of ER- $\alpha$ and PR did not change. While the function of ER- $\beta$ in breast cancer cells remains controversial, it has been implicated as a negative regulator of cell growth and proliferation (26-29). A recent report has also indicated a role for ER- $\beta$ in blocking angiogenesis in T47-D by reducing VEGF (30) and ER-B has been shown to interact with the VEGF promoter (31). In addition, it has been shown that ER- $\beta$ negatively impacts the 
proliferative effects of ER- $\alpha$ by altering ER- $\alpha$ mediated gene expression in breast cancer cells $(32,33)$. Thus it is possible that increased expression of ER- $\beta$ during PRIMA- 1 treatment may lead to loss of progression of breast cancer cells though the cells are continuously exposed to estrogen. The mechanism by which PRIMA-1 stimulates expression of ER- $\beta$ in breast cancer cells, and how such an increase influences ER- $\alpha$ mediated proliferative response remains to be explored.

In conclusion, our results show that the p53 pathway negatively regulates growth of T47-D cells in vivo and in vitro, and that activation of the p53 pathway suppresses the transformed phenotype of these cells. In cell culture, this effect is observed in the presence of estradiol. An important outcome of this study is that endogenous mtp53 can be re-activated by small molecule p53 activators such as PRIMA-1, and such compounds may have therapeutic potential for preventing or treating at least a subset of human breast tumors.

\section{Acknowledgements}

This research was supported by NIH grant CA-86916; Dept of Defense Breast Cancer Research Program W81XWH-051-0416; PDF0600723 and BCTR0600704 from Susan G Komen Breast Cancer Foundation, and by funds from the Research Animal Diagnostic Laboratory. We would like to thank Dr Xinbin Chen from the University of Alabama for the p53 expression vector. We would also like to thank Ms. Jennifer Schnell and Ms. Jill Gruenkemeyer for excellent technical assistance. S.M.H. is the Zalk Missouri Professor of Tumor Angiogenesis.

\section{References}

1. Levine AJ: p53, the cellular gatekeeper for growth and division. Cell 88: 323-331, 1997.

2. Barnes DM and Camplejohn RS: p53, apoptosis, and breast cancer. J Mammary Gland Biol Neoplasia 1: 163-175, 1996.

3. Nigro JM, Baker SJ, Preisinger AC, et al: Mutations in the p53 gene occur in diverse human tumor types. Nature 342: 705-708, 1989.

4. Bartek J, Bartkova J, Vojtesek B, Staskova Z, Rejthar A, Kovarik J and Lane DP: Patterns of expression of the p53 tumour suppressor in human breast tissues and tumors in situ and in vitro. Int J Cancer 46: 1839-1844, 1990.

5. Gewirtz DA: Growth arrest and cell death in the breast tumor cell in response to ionizing radiation and chemotherapeutic agents which induce DNA damage. Breast Cancer Res Treat 62: 223-235, 2000.

6. Bottini A, Berruti A, Bersiga A, et al: p53 but not bcl-2 immunostaining is predictive of poor clinical complete response to primary chemotherapy in breast cancer patients. Clin Cancer Res 6: 2751-2758, 2000.

7. Rahko E, Blanco G, Soini Y, Bloigu R and Jukkola A: A mutant TP53 gene status is associated with a poor prognosis and anthracycline-resistance in breast cancer patients. Eur J Cancer 39: 447-453, 2003.

8. Muller M, Wilder S, Bannasch D, et al: p53 activates the CD95 (APO-1/Fas) gene in response to DNA damage by anticancer drugs. J Exp Med 188: 2033-2045, 1998.

9. Bykov VJ, Issaeva N, Shilov A, et al: Restoration of the tumor suppressor function to mutant p53 by a low-molecular-weight compound. Nat Med 8: 282-288, 2002.

10. Liang Y, Wu J and Hyder SM: p53-dependent inhibition of progestin-induced VEGF expression in human breast cancer cells. J Steroid Biochem Mol Biol 93: 173-182, 2005.

11. Folkman J: Angiogenesis in cancer, vascular, rheumatoid and other disease. Nat Med 1: 27-31, 1995.
12. Bergers $\mathrm{G}$ and Benjamin LE: Tumorigenesis and the angiogenic switch. Nat Rev Cancer 3: 401-410, 2003.

13. Rubenstein LV, Shoemaker RH, Paull KD, et al: Comparison of in vitro anticancer-drug-screening data generated with a tetrazolium assay versus a protein assay against a diverse panel of human tumor cell lines. J Natl Cancer Inst 82: 1113-1118, 1990.

14. Skehan P, Storeng R, Scudiero D, et al: New colorimetric cytotoxicity assay for anti-cancer-drug screening. J Natl Cancer Inst 82: 1107-1112, 1990.

15. El Etreby MF and Liang Y: Effect of antiprogestins and tamoxifen on growth inhibition of MCF-7 human breast cancer cells in nude mice. Breast Cancer Res Treat 49: 109-117, 1998.

16. Lewis JS, Cheng D and Jordan VC: Targeting oestrogen to kill the cancer but not the patient. Br J Cancer 90: 944-949, 2004.

17. Dummer R, Bergh J, Karlsson Y, et al: Biological activity and safety of adenoviral vector-expressed wild-type p53 after intratumoral injection in melanoma and breast cancer patients with p53-overexpressing tumors. Cancer Gene Ther 7: 1069-1076, 2000 .

18. Cristofanilli M, Krishnamurthy S, Guerra L, et al: A nonreplicating adenoviral vector that contains the wild-type p53 transgene combined with chemotherapy for primary breast cancer: safety, efficacy, and biologic activity of a novel genetherapy approach. Cancer 107: 935-944, 2006.

19. Yu CL, Driggers P, Barrera-Hernandez G, Nunez SB, Segars JH and Cheng S: The tumor suppressor p53 is a negative regulator of estrogen receptor signaling pathways. Biochem Biophys Res Commun 239: 617-620, 1997.

20. Liu G, Schwartz JA and Brooks SC: p53 down-regulates ERresponsive genes by interfering with the binding of ER to ERE. Biochem Biophys Res Commun 264: 359-364, 1999.

21. Liu W, Konduri SD, Bansal S, et al: Estrogen receptor-alpha binds p53 tumor suppressor protein directly and represses its function. J Biol Chem 281: 9837-9840, 2006.

22. Diel P: Tissue-specific estrogenic response and molecular mechanisms. Toxicol Lett 127: 217-224, 2002.

23. Liang Y, Brekken RA and Hyder SM: VEGF induces proliferation of breast cancer cells and counteracts the anti-proliferative activity of anti-hormones. End Related Cancer 13: 905-919, 2006.

24. Mukhopadhyay D, Tsiokas L and Sukhatme VP: A Wild-type p53 and v-Src exert opposing influences on human vascular endothelial growth factor gene expression. Cancer Res 55: 6161-6165, 1995

25. Nishizaki M, Fujiwara T, Tanida T, et al: Recombinant adenovirus expressing wild-type p53 is antiangiogenic: a proposed mechanism for bystander effect. Clin Cancer Res 5: 1015-1023, 1995.

26. Gustafsson JA and Warner M: Estrogen receptor beta in the breast: role in estrogen responsiveness and development of breast cancer. J Steroid Biochem Mol Biol 74: 245-248, 2000.

27. Matthews J and Gustafsson JA: Estrogen signaling: a subtle balance between ER alpha and ER beta. Mol Interv 3: 281-292, 2003.

28. Pearce ST and Jordan VC: The biological role of estrogen receptors alpha and beta in cancer. Crit Rev Oncol Hematol 50: 3-22, 2004.

29. Bardin A, Boulle N, Lazennec G, Vignon F and Pujol P: Loss of ERbeta expression as a common step in estrogen-dependent tumor progression. Endocr Relat Cancer 1: 537-551, 2004.

30. Hartman J, Lindberg K, Morani A, Inzunza J, Strom A and Gustafsson JA: Estrogen receptor beta inhibits angiogenesis and growth of T47D breast cancer xenografts. Cancer Res 66: 11207-11213, 2006.

31. Hyder SM, Nawaz Z, Chiappetta C and Stancel GM: Identification of functional estrogen response elements in the gene coding for the potent angiogenic factor vascular endothelial growth factor. Cancer Res 60: 3183-3190, 2000.

32. Hall JM and McDonnell DP: The estrogen receptor beta-isoform (ERbeta) of the human estrogen receptor modulates ERalpha transcriptional activity and is a key regulator of the cellular response to estrogens and antiestrogens. Endocrinology 140: 5566-5578, 1999.

33. Chang EC, Frasor J, Komm B and Katzenellenbogen BS: Impact of estrogen receptor beta on gene networks regulated by estrogen receptor alpha in breast cancer cells. Endocrinology 147: 4831-4842, 2006. 